MATHEMATICS OF COMPUTATION

Volume 73, Number 245, Pages 259-277

S 0025-5718(03)01531-X

Article electronically published on April 28, 2003

\title{
OPTIMAL QUADRATURE FOR HAAR WAVELET SPACES
}

\author{
STEFAN HEINRICH, FRED J. HICKERNELL, AND RONG-XIAN YUE
}

\begin{abstract}
This article considers the error of the scrambled equidistribution quadrature rules in the worst-case, random-case, and average-case settings. The underlying space of integrands is a Hilbert space of multidimensional Haar wavelet series, $\mathcal{H}_{\text {wav }}$. The asymptotic orders of the errors are derived for the case of the scrambled $(\lambda, t, m, s)$-nets and $(t, s)$-sequences. These rules are shown to have the best asymptotic convergence rates for any random quadrature rule for the space of integrands $\mathcal{H}_{\text {wav }}$.
\end{abstract}

\section{INTRODUCTION}

Base $b$ scrambling quadrature, proposed by Owen [Owe95], is a hybrid of Monte Carlo and quasi-Monte Carlo methods. One starts with a low discrepancy set or sequence and randomly permutes the digits of the points. When applied to a $(t, m, s)$-net or a $(t, s)$-sequence Nie92, Lar98, the result is a new net or sequence with the same $t$-value. Since each scrambled point is uniformly distributed over the unit cube, the resulting scrambled quadrature rule is unbiased. Moreover, the quadrature error may be estimated by performing a number of replications of the scrambled rule. Furthermore, there is a central limit theorem for $(0, m, s)$-nets as $m$ tends to infinity [Loh01.

The purpose of this article is to show that scrambled net quadrature rules are optimal for spaces of functions that are multidimensional Haar wavelet series. This means that the asymptotic convergence rate for scrambled net quadrature is the highest possible for any quadrature rule. This result holds for worst-case, randomcase and average-case error analyses, and the asymptotic convergence rates are obtained explicitly.

The integration problem studied here is integration over the $s$-dimensional unit cube:

$$
I(f)=\int_{[0,1)^{s}} f(\mathbf{x}) d \mathbf{x} .
$$

Quadrature rules to approximate this integral take the form

$$
Q\left(f ; P,\left\{w_{i}\right\}\right)=\sum_{i=1}^{n} w_{i} f\left(\mathbf{x}_{i}\right)
$$

Received by the editor July 9, 2001 and, in revised form, May 13, 2002.

2000 Mathematics Subject Classification. Primary 65C05, 65D30.

Key words and phrases. Quasi-Monte Carlo methods, Monte Carlo methods, high dimensional integration, lower bounds.

This work was partially supported by a Hong Kong Research Grants Council grant HKBU/2030/99P, by Hong Kong Baptist University grant FRG/97-98/II-99, by Shanghai NSF Grant 00JC14057, and by Shanghai Higher Education STF grant 01D01-1.

(C)2003 American Mathematical Society 
for some set of nodes $P=\left\{\mathbf{x}_{1}, \ldots, \mathbf{x}_{n}\right\} \subset[0,1)^{s}$ and some set of weights $\left\{w_{i}\right\}=$ $\left\{w_{1}, \ldots, w_{n}\right\}$. Quasi-Monte Carlo quadrature methods choose $P$ to be a set of points evenly distributed over the integration domain, and $w_{i}=n^{-1}$ for all $i$.

The quality of a quadrature rule can be assessed by a worst-case, random-case, or average-case error analysis HW01. Let $\mathcal{H}$ denote some separable Hilbert space of measurable functions, and let $\mathcal{B}$ be the unit ball in $\mathcal{H}$, i.e., $\mathcal{B}=\{f \in \mathcal{H}$ : $\left.\|f\|_{\mathcal{H}} \leq 1\right\}$. The quadrature error for a specific integrand in $\mathcal{H}$ and a specific quadrature rule $Q$ is given by $\operatorname{Err}(f ; Q)=I(f)-Q\left(f ; P,\left\{w_{i}\right\}\right)$. Suppose that $Q$ is random, i.e., the nodes, weights, and number of function evaluations are all chosen randomly. Specifically, let $Q$ be chosen from some sample space $\mathcal{Q}_{n}$ equipped with some probability distribution $\mu$, where the average number of function evaluations is $n$. (Deterministic quadrature rules are the case where $\mathcal{Q}_{n}$ has a single element.) It is assumed that any particular quadrature rule makes at least one function evaluation, so that (11) makes sense. In the average case we assume that $\mathcal{H}$ is equipped with a probability measure such that $E\|f\|_{\mathcal{H}}^{2}=1$.

The worst-case, random-case, and average-case error criteria for a given Hilbert space of integrands and type of rules are:

$$
\begin{array}{rlrl}
\text { worst-case: } & e^{\mathrm{w}}\left(\mathcal{H} ; \mathcal{Q}_{n}\right): & =\operatorname{rms}_{Q \in \mathcal{Q}_{n}} \sup _{f \in \mathcal{B}}|\operatorname{Err}(f ; Q)|, \\
\text { random-case: } & e^{\mathrm{r}}\left(\mathcal{H} ; \mathcal{Q}_{n}\right):=\sup _{f \in \mathcal{B}} \operatorname{rms}_{Q \in \mathcal{Q}_{n}}|\operatorname{Err}(f ; Q)|, \\
\text { average-case: } & e^{\mathrm{a}}\left(\mathcal{H} ; \mathcal{Q}_{n}\right):=\operatorname{rms}_{Q \in \mathcal{Q}_{n}} \operatorname{rms}_{f \in \mathcal{H}}|\operatorname{Err}(f ; Q)| .
\end{array}
$$

The operator rms means root mean square (or the respective expression with the upper integral if the function to which rms is applied is not measurable).

The optimal error criteria for a Hilbert space are defined as the infima of the above with respect to all possible quadrature rules:

$$
e^{\mathrm{x}}(\mathcal{H}, n):=\inf _{\mathcal{Q}_{n}} e^{\mathrm{x}}\left(\mathcal{H} ; \mathcal{Q}_{n}\right), \quad \mathrm{x} \in\{\mathrm{w}, \mathrm{r}, \mathrm{a}\} .
$$

A sequence of random quadrature rules $\left(\mathcal{Q}_{n_{m}}\right)_{m=0,1,2, \ldots}$ is said to be optimal if it has the same asymptotic order as the best possible quadrature rules. Specifically, one has worst-case, random-case, and average-case optimality, respectively, if there exists some nonzero constant $C$ independent of $n$ such that for all $n=1,2, \ldots$,

$$
\min _{n_{m} \leq n} e^{\mathrm{x}}\left(\mathcal{H} ; \mathcal{Q}_{n_{m}}\right) \leq C e^{\mathrm{x}}(\mathcal{H}, n),
$$

where $\mathrm{x}=\mathrm{w}, \mathrm{r}, \mathrm{a}$, respectively. It is possible for a sequence of quadrature rules to be optimal for one of the above criteria and not for the others.

This article is concerned with Haar wavelet spaces, $\mathcal{H}_{\text {wav }}$, defined in Section 2 and quadrature rules based on scrambled $(\lambda, t, m, s)$-nets and $(t, s)$-sequences in base $b$ and weights all chosen to be $w_{i}=1 / n$. It is shown in Corollary 11] that scrambled net rules, $\mathcal{Q}_{\mathrm{sc}, n}$, are optimal for integrating functions in $\mathcal{H}_{\text {wav }}$ in the worst, random, and average cases. This is done by showing that for some constants $C_{\mathrm{up}}$ and $C_{\mathrm{lo}}$ and some function $h^{\mathrm{x}}(n)$,

$$
\min _{n_{m} \leq n} e^{\mathrm{x}}\left(\mathcal{H}_{\mathrm{wav}} ; \mathcal{Q}_{\mathrm{sc}, n_{m}}\right) \leq C_{\mathrm{up}} h^{\mathrm{x}}(n), \quad n=1,2, \ldots,
$$

in Theorem 8, and

$$
C_{\mathrm{lo}} h^{\mathrm{x}}(n) \leq e^{\mathrm{x}}\left(\mathcal{H}_{\text {wav }}, n\right), \quad n=1,2, \ldots
$$


in Theorems 9 and 10. Here $\mathrm{x} \in\{\mathrm{w}, \mathrm{r}, \mathrm{a}\}$ again. The formula for $h^{\mathrm{x}}(n)$ is found explicitly, so the asymptotic convergence rate is known. Equation (44) can be derived by applying the results of Hic96, HH99, HY00, for worst-case and average-case error and the results of Owe97a, Owe97b, Owe98, Yue99, YM99 for random-case error. The problem of tractability (unbounded dimension) is not considered here, but has been studied by YH01. Obtaining lower bounds relies on constructing test functions (or systems of such, with a measure on them, in the random-case) that cannot be integrated too well by any quadrature rule. This approach is explained in Nov88] and [Hei93].

There are a couple of reasons for considering the Hilbert space $\mathcal{H}_{\text {wav }}$. First, the technical details of computing the upper bounds in (4) for scrambled nets are relatively easy for this space compared to other spaces. In other words, $\mathcal{H}_{\text {wav }}$ seems to be the natural space for studying scrambled net quadrature. Second, it is known that for many other spaces $\mathcal{H}$, computing $e^{\mathrm{w}}\left(\mathcal{H} ; \mathcal{Q}_{\mathrm{sc}, n}\right)$ is equivalent to computing $e^{\mathrm{w}}\left(\mathcal{H}_{\mathrm{wav}} ; \mathcal{Q}_{\mathrm{sc}, n}\right)$ HY00. A similar relationship holds for average-case error analysis, but unfortunately the situation is not so simple for random-case error analysis.

\section{FunCtion SPACES SPANNED By HaAR WAVELETS}

This section defines the multidimensional spaces of Haar wavelets that are used in Owe97a and elsewhere. Let $b$ be an integer greater than one that will denote the base of the Haar wavelets and the $(t, s)$-sequences. Define the univariate basic wavelet functions,

$$
\psi_{\gamma}(x)=b^{1 / 2} 1_{\lfloor b x\rfloor=\gamma}-b^{-1 / 2} 1_{\lfloor x\rfloor=0}, \quad \gamma=0,1, \ldots, b-1,
$$

where $1_{\{\cdot\}}$ denotes the characteristic function, and $\lfloor x\rfloor$ denotes the floor function of $x$ or greatest integer less than or equal to $x$. For integers $0 \leq \kappa$ and $0 \leq \tau<b^{\kappa}$ the dilated and translated versions of the above functions are the following univariate wavelets:

$$
\psi_{\kappa \tau \gamma}(x)=b^{k / 2} \psi_{\gamma}\left(b^{\kappa} x-\tau\right)=b^{(k-1) / 2}\left(b 1_{\left\lfloor b^{k+1} x\right\rfloor=b \tau+\gamma}-1_{\left\lfloor b^{k} x\right\rfloor=\tau}\right) .
$$

For each subset $u$ of the coordinate axes $\{1, \ldots, s\}$, let $|u|$ denote the cardinality of $u$, and let $\bar{u}$ denote the complement, $\{1, \ldots, s\}-u$. For each $r \in u$ let $\kappa_{r}, \tau_{r}$ and $\gamma_{r}$ be integers with $\kappa_{r} \geq 0,0 \leq \tau_{r}<b^{\kappa_{r}}$ and $0 \leq \gamma_{r}<b$. Define the vectors $\boldsymbol{\kappa}=\left(\kappa_{r}\right)_{r \in u}, \boldsymbol{\tau}=\left(\tau_{r}\right)_{r \in u}$, and $\boldsymbol{\gamma}=\left(\gamma_{r}\right)_{r \in u}$. Let $\psi_{u \kappa \tau \gamma}$ be a product over $r \in u$ of the dilated and translated wavelets, i.e.,

$$
\begin{aligned}
\psi_{u \kappa \tau \gamma}(\mathbf{x}) & :=\prod_{r \in u} \psi_{\kappa_{r} \tau_{r} \gamma_{r}}\left(x_{r}\right) \\
& =b^{(|\boldsymbol{\kappa}|-|u|) / 2} \prod_{r \in u}\left(b 1_{\left\lfloor b^{\kappa_{r}+1} x_{r}\right\rfloor=b \tau_{r}+\gamma_{r}}-1_{\left\lfloor b^{\kappa_{r}} x_{r}\right\rfloor=\tau_{r}}\right),
\end{aligned}
$$

where $|\boldsymbol{\kappa}|=\sum_{r \in u} \kappa_{r}$. For $u=\emptyset$ we take by convention $\psi_{u \boldsymbol{\kappa} \tau \boldsymbol{\gamma}}(\mathbf{x})=\psi_{\emptyset}(\mathbf{x})=1$. The wavelets defined above are not orthogonal nor linearly independent, but they are nearly so. As observed in Owe97a,

$$
\begin{gathered}
\sum_{\gamma_{r}=0}^{b-1} \psi_{u \kappa \boldsymbol{\tau} \gamma}(\mathbf{x})=0, \quad \forall u \neq \emptyset, \forall \boldsymbol{\kappa}, \boldsymbol{\tau}, \forall r \in u, \forall \gamma_{r^{\prime}} \text { with } r \neq r^{\prime} \in u, \\
\int_{[0,1)^{s}} \psi_{u \kappa \boldsymbol{\tau} \boldsymbol{\gamma}}(\mathbf{x}) \psi_{u^{\prime} \boldsymbol{\kappa}^{\prime} \boldsymbol{\tau}^{\prime} \boldsymbol{\gamma}^{\prime}}(\mathbf{x}) d \mathbf{x}=\delta_{u u^{\prime}} \delta_{\boldsymbol{\kappa} \boldsymbol{\kappa}^{\prime}} \delta_{\boldsymbol{\tau} \boldsymbol{\tau}^{\prime}} \prod_{r \in u}\left(\delta_{\gamma_{r} \gamma_{r}^{\prime}}-b^{-1}\right),
\end{gathered}
$$


where $\delta$ is the Kronecker delta function.

From [Owe97a] any function $f \in \mathcal{L}_{2}\left([0,1)^{s}\right)$ can be represented as

$$
f=\sum_{u, \kappa, \boldsymbol{\tau}, \boldsymbol{\gamma}} \hat{f}_{u \kappa \tau \gamma} \psi_{u \kappa \tau \gamma}
$$

where the coefficients $\hat{f}_{u \kappa \tau \gamma}$ are given by

$$
\hat{f}_{u \kappa \tau \gamma}:=\int_{[0,1)^{s}} f(\mathbf{x}) \psi_{u \kappa \tau \gamma}(\mathbf{x}) d \mathbf{x} .
$$

The coefficients satisfy

$$
\sum_{u, \kappa, \boldsymbol{\tau}, \boldsymbol{\gamma}} \hat{f}_{u \kappa \tau \gamma}^{2}<\infty
$$

and the series (8) converges in $\mathcal{L}_{2}\left([0,1)^{s}\right)$. Note that $\hat{f}_{\emptyset}$ is simply $I(f)$, the integral of the function. Because the wavelets are not linearly independent, they do not form a basis, and the series expression in (86) for $f$ is not unique. By (6) and (9) it follows that

$$
\sum_{\gamma_{r}=0}^{b-1} \hat{f}_{u \kappa \tau \gamma}=0, \quad \forall u \neq \emptyset, \forall \boldsymbol{\kappa}, \boldsymbol{\tau}, \forall r \in u, \forall \gamma_{r^{\prime}} \text { with } r \neq r^{\prime} \in u .
$$

This condition ensures uniqueness of the representation in (88) in the sense that whenever

$$
f=\sum_{u, \kappa, \tau, \gamma} c_{u \kappa \tau \gamma} \psi_{u \kappa \tau \gamma}
$$

where the $c_{u \kappa \tau \gamma}$ satisfy

$$
\sum_{u, \kappa, \tau, \gamma} c_{u \kappa \tau \gamma}^{2}<\infty
$$

and

$$
\sum_{\gamma_{r}=0}^{b-1} c_{u \kappa \tau \gamma}=0 \quad \forall u \neq \emptyset, \forall \boldsymbol{\kappa}, \boldsymbol{\tau}, \forall r \in u, \forall \gamma_{r^{\prime}} \text { with } r \neq r^{\prime} \in u,
$$

it follows that

$$
c_{u \kappa \tau \gamma}=\hat{f}_{u \kappa \tau \gamma} \quad \forall u, \kappa, \tau, \gamma .
$$

The space of integrands, $\mathcal{H}_{\text {wav }}$, considered in this article consists of all wavelet series whose coefficients converge to zero quickly enough. Let $\omega_{u \kappa}$ denote some positive scalars defined for all $u$ and $\boldsymbol{\kappa}$, satisfying

$$
\sup _{u \kappa} \omega_{u \kappa}<\infty .
$$

Define

$$
\mathcal{H}_{\text {wav }}=\left\{f \in \mathcal{L}_{2}\left([0,1)^{s}\right): \sum_{u, \kappa, \boldsymbol{\tau}, \boldsymbol{\gamma}} \omega_{u \kappa}^{-1} \hat{f}_{u \kappa \tau \gamma}^{2}<\infty\right\},
$$

endowed with the inner product

$$
\langle f, g\rangle_{\mathcal{H}_{\mathrm{wav}}}=\sum_{u, \kappa, \boldsymbol{\tau}, \boldsymbol{\gamma}} \omega_{u \kappa}^{-1} \hat{f}_{u \kappa \tau \gamma} \hat{g}_{u \kappa \tau \gamma}
$$


Furthermore, define the scaled wavelets $\psi_{u \kappa \tau \gamma}^{\omega}(\mathbf{x})=\omega_{u \kappa}^{1 / 2} \psi_{u \kappa \tau \gamma}(\mathbf{x})$, and let $\hat{f}_{u \kappa \tau \gamma}^{\omega}=$ $\omega_{u \kappa}^{-1 / 2} \hat{f}_{u \kappa \tau \gamma}$. Then integrands in $\mathcal{H}_{\text {wav }}$ can be represented as

$$
f=\sum_{u, \kappa, \tau, \gamma} \hat{f}_{u \kappa \tau \gamma}^{\omega} \psi_{u \kappa \tau \gamma}^{\omega}=\hat{\mathbf{f}}_{\omega}^{T} \boldsymbol{\psi}_{\omega}
$$

where $\hat{\mathbf{f}}_{\omega}$ is the column vector of the coefficients $\hat{f}_{u \kappa \tau \gamma}^{\omega}$, and $\boldsymbol{\psi}_{\omega}$ is the column vector of the basis functions $\psi_{u \kappa \tau \gamma}^{\omega}$. The inner product and norm of $\mathcal{H}_{\text {wav }}$ can be expressed in terms of the scalar product and $\mathcal{L}_{2}$-norm of the coefficient vectors as follows:

$$
\langle f, g\rangle_{\mathcal{H}_{\text {wav }}}=\left\langle\hat{\mathbf{f}}_{\omega}, \hat{\mathbf{g}}_{\omega}\right\rangle_{2}=\hat{\mathbf{f}}_{\omega}^{T} \hat{\mathbf{g}}_{\omega}, \quad\|f\|_{\mathcal{H}_{\text {wav }}}=\left\|\hat{\mathbf{f}}_{\omega}\right\|_{2}=\left(\hat{\mathbf{f}}_{\omega}^{T} \hat{\mathbf{f}}_{\omega}\right)^{1 / 2} .
$$

\section{Formulae For the ERROR CRITERIA UNDER SCRAMBlED QUADRATURE}

Now we turn to the problem of computing the error criteria defined in (2). The derivations in this section follow the arguments given by [HW01. A key ingredient in this analysis is the $\infty \times \infty$ matrix whose elements are the expectations of products of integration errors of any two wavelet functions by a randomized quadrature. Define

$$
\boldsymbol{\Lambda}:=E_{Q \in \mathcal{Q}_{n}}\left[\operatorname{Err}\left(\boldsymbol{\psi}_{\omega} ; Q\right) \operatorname{Err}\left(\boldsymbol{\psi}_{\omega} ; Q\right)^{T}\right] .
$$

For the worst-case analysis one must assume that the quadrature rule is welldefined for any choice of sample points $P$. This means that function evaluations must be bounded linear functionals on the Hilbert space $\mathcal{H}_{\text {wav }}$. To ensure this it is assumed that

$$
\sum_{u, \boldsymbol{\kappa}, \boldsymbol{\tau}, \boldsymbol{\gamma}}\left|\psi_{u \kappa \tau \gamma}^{\omega}(\mathbf{x})\right|^{2}=\sum_{u \kappa}(b-1)^{|u|} b^{|\kappa|} \omega_{u \kappa}<\infty,
$$

which implies that the series defining the integrands in $\mathcal{H}_{\text {wav }}$ is pointwise absolutely convergent.

For the random-case and average-case error analyses it is only necessary that the integrand be in $\mathcal{L}_{2}\left([0,1)^{s}\right)$, which is guaranteed by the definition of $\mathcal{H}_{\text {wav }}$. For the average-case error analysis the integrand $f$ is assumed to be defined in terms of random series coefficients $\hat{f}_{u \kappa \tau \gamma}^{\omega}$. Specifically the vector $\hat{\mathbf{f}}_{\omega}$ of series coefficients is assumed to have zero mean and covariance matrix $\boldsymbol{\Sigma}$. In this article this matrix is assumed to be block diagonal with blocks of the form

$$
\boldsymbol{\Sigma}_{u \kappa \tau}=\sigma_{u \kappa}\left[\otimes^{|u|} \mathbf{H}_{b}\right]
$$

where the $b \times b$ matrix $\mathbf{H}_{b}$ is defined as

$$
\mathbf{H}_{b}=\mathbf{I}_{b}-b^{-1} \mathbf{1}_{b} \mathbf{1}_{b}^{T},
$$

and $\otimes^{|u|} \mathbf{H}_{b}$ means taking the Kronecker product of $\mathbf{H}_{b}$ with itself $|u|$ times. This form of the covariance is consistent with condition (10). As noted in [HW01,

$$
E_{f \in \mathcal{H}_{\text {wav }}}\|f\|_{\mathcal{H}_{\text {wav }}}^{2}=E_{f \in \mathcal{H}_{\text {wav }}}\langle f, f\rangle_{\mathcal{H}_{\text {wav }}}=E_{f \in \mathcal{H}_{\text {wav }}}\left\langle\hat{\mathbf{f}}_{\omega}, \hat{\mathbf{f}}_{\omega}\right\rangle_{2}=\operatorname{trace}(\boldsymbol{\Sigma}) .
$$

In order to ensure that this quantity is unity, the positive scalars $\sigma_{u \kappa}$ must satisfy the following condition:

$$
\sum_{u \kappa}(b-1)^{|u|} b^{|\kappa|} \sigma_{u \kappa}=1
$$


Theorem 1. Consider the case of random quadrature rules applied to integrands that take the form of wavelet series. The three error criteria defined in (2) are given by

$$
\begin{aligned}
e^{\mathrm{w}}\left(\mathcal{H}_{\text {wav }} ; \mathcal{Q}_{n}\right) & =\sqrt{\operatorname{trace}(\boldsymbol{\Lambda})}, \text { assuming (14), } \\
e^{\mathrm{r}}\left(\mathcal{H}_{\text {wav }} ; \mathcal{Q}_{n}\right) & =\sqrt{\rho(\boldsymbol{\Lambda})}, \\
e^{\mathrm{a}}\left(\mathcal{H}_{\text {wav }} ; \mathcal{Q}_{n}\right) & =\sqrt{\operatorname{trace}(\boldsymbol{\Lambda} \boldsymbol{\Sigma})}, \text { assuming (17). }
\end{aligned}
$$

Proof. To prove (18a), first define the vector $\hat{\boldsymbol{\xi}}_{Q, \omega}=\operatorname{Err}\left(\boldsymbol{\psi}_{\omega} ; Q\right)$ and the function $\xi_{Q}(\mathbf{x})=\hat{\boldsymbol{\xi}}_{Q, \omega}^{T} \boldsymbol{\psi}_{\omega}(\mathbf{x})$ for any quadrature rule $Q$. By assumption (14) it follows that the vector $\hat{\boldsymbol{\xi}}_{Q, \omega}$ has finite $\ell_{2}$-norm, and by (6) it follows that $\hat{\boldsymbol{\xi}}_{Q, \omega}$ satisfies (10). Thus, $\xi_{Q} \in \mathcal{H}_{\text {wav }}$. For any integrand $f=\hat{\mathbf{f}}_{\omega}^{T} \boldsymbol{\psi}_{\omega} \in \mathcal{H}_{\text {wav }}$, its quadrature error may be expressed as the inner product of $f$ with $\xi_{Q}$ :

$$
\operatorname{Err}(f ; Q)=\hat{\mathbf{f}}_{\omega}^{T} \operatorname{Err}\left(\boldsymbol{\psi}_{\omega} ; Q\right)=\left\langle f, \xi_{Q}\right\rangle_{\mathcal{H}_{\text {wav }}} .
$$

Let $\mathcal{B}_{\text {wav }}$ denote the unit ball in $\mathcal{H}_{\text {wav }}$. Then applying the Cauchy-Schwarz inequality yields a tight worst-case error bound for this quadrature rule:

$$
\sup _{f \in \mathcal{B}_{\text {wav }}}|\operatorname{Err}(f ; Q)|=\sup _{\|f\|_{\mathcal{H}_{\text {wav }} \leq 1}}\left|\left\langle f, \xi_{Q}\right\rangle_{\mathcal{H}_{\text {wav }}}\right|=\left\|\xi_{Q}\right\|_{\mathcal{H}_{\text {wav }}}
$$

The quantity $\left\|\xi_{Q}\right\|_{\mathcal{H}_{\text {wav }}}$ is often called the discrepancy. Note that the discrepancy may be expressed as the trace of a matrix:

$$
\begin{aligned}
\left\|\xi_{Q}\right\|_{\mathcal{H}_{\text {wav }}}^{2} & =\left\|\operatorname{Err}\left(\boldsymbol{\psi}_{\omega} ; Q\right)\right\|_{2}^{2}=\operatorname{Err}\left(\boldsymbol{\psi}_{\omega} ; Q\right)^{T} \operatorname{Err}\left(\boldsymbol{\psi}_{\omega} ; Q\right) \\
& =\operatorname{trace}\left[\operatorname{Err}\left(\boldsymbol{\psi}_{\omega} ; Q\right) \operatorname{Err}\left(\boldsymbol{\psi}_{\omega} ; Q\right)^{T}\right] .
\end{aligned}
$$

It follows from the definitions in (2a) and (13) that the worst-case error criterion is $e^{\mathrm{w}}\left(\mathcal{H}_{\text {wav }} ; \mathcal{Q}_{n}\right)=\sqrt{\operatorname{trace}(\boldsymbol{\Lambda})}$.

To prove (18b), it is required to compute the root mean square error in integrating an arbitrary integrand $f(\mathbf{x})=\hat{\mathbf{f}}_{\omega}^{T} \boldsymbol{\psi}_{\omega}(\mathbf{x}) \in \mathcal{H}_{\text {wav }}$. From the definition in (13) it follows that

$$
\operatorname{rms}_{Q \in \mathcal{Q}_{n}}|\operatorname{Err}(f ; Q)|=\sqrt{\hat{\mathbf{f}}_{\omega}^{T} \boldsymbol{\Lambda} \hat{\mathbf{f}}_{\omega}} .
$$

The random-case error criterion is then given by

$$
e^{\mathrm{r}}\left(\mathcal{H}_{\text {wav }} ; \mathcal{Q}_{n}\right)=\sup _{f \in \mathcal{B}_{\text {wav }}} \operatorname{rms}_{Q \in \mathcal{Q}_{n}}|\operatorname{Err}(f ; Q)|=\sup _{\substack{\left\|\hat{\mathbf{f}}_{\omega}\right\|_{2} \leq 1 \\ \hat{\mathbf{f}}_{\omega} \text { satisfies 10 }}} \sqrt{\hat{\mathbf{f}}_{\omega}^{T} \boldsymbol{\Lambda} \hat{\mathbf{f}}_{\omega}} .
$$

Without condition (10) this would be simply $\sqrt{\rho(\boldsymbol{\Lambda})}$, the square root of the spectral radius (largest absolute eigenvalue) of $\boldsymbol{\Lambda}$.

In fact, $e^{\mathrm{r}}\left(\mathcal{H}_{\mathrm{wav}} ; \mathcal{Q}_{n}\right)=\sqrt{\rho(\boldsymbol{\Lambda})}$ even with condition (10). To prove this, we show that the eigenvector $\mathbf{v}$ of $\boldsymbol{\Lambda}$ corresponding to the maximum eigenvalue satisfies constraint (10). By definition $\boldsymbol{\Lambda}$ is a symmetric, nonnegative definite matrix. Without loss of generality, assume that the largest eigenvalue is positive. For any fixed $u, \boldsymbol{\kappa}, \boldsymbol{\tau}, r \in u$, and $\boldsymbol{\gamma}_{u-\{r\}}$, let $\mathbf{w}$ be the vector whose $u \boldsymbol{\kappa} \tau \boldsymbol{\gamma}$ elements are all one for $\gamma_{r}=0, \ldots, b-1$ and zero otherwise. By the definition of $\boldsymbol{\Lambda}$ and (6) it follows that this $\mathbf{w}$ is an eigenvector of $\boldsymbol{\Lambda}$ with zero eigenvalue, i.e., $\boldsymbol{\Lambda} \mathbf{w}=\mathbf{0}$. Since $\boldsymbol{\Lambda}$ is symmetric, $\mathbf{w}^{T} \mathbf{v}=0$, which implies that $\mathbf{v}$ satisfies (10). 
For the average-case error criterion defined in (18c), note that the order of the two root mean squares can be exchanged. Starting from (19) and applying the standard formulas for expectations of quadratic forms of random variables yields

$$
\begin{aligned}
e^{\mathrm{a}}\left(\mathcal{H}_{\text {wav }} ; \mathcal{Q}_{n}\right) & =\sqrt{E_{f \in \mathcal{H}_{\text {wav }}\left|\operatorname{rms}_{Q \in \mathcal{Q}_{n}}\right| \operatorname{Err}(f ; Q)||^{2}}} \\
& =\sqrt{E_{f \in \mathcal{H}_{\text {wav }}}\left[\hat{\mathbf{f}}_{\omega}^{T} \boldsymbol{\Lambda}^{\mathrm{sc}} \hat{\mathbf{f}}_{\omega}\right]}=\sqrt{\operatorname{trace}(\boldsymbol{\Lambda} \boldsymbol{\Sigma})}
\end{aligned}
$$

From Theorem 1 it is clear that

$$
e^{\mathrm{w}}\left(\mathcal{H}_{\text {wav }} ; \mathcal{Q}_{\mathrm{sc}, n}\right) \geq e^{\mathrm{r}}\left(\mathcal{H}_{\text {wav }} ; \mathcal{Q}_{\mathrm{sc}, n}\right) \geq e^{\mathrm{a}}\left(\mathcal{H}_{\text {wav }} ; \mathcal{Q}_{\mathrm{sc}, n}\right)
$$

Since the matrix $\boldsymbol{\Lambda}$ is positive semi-definite, its eigenvalues are all nonnegative, and so its trace must be at least as large as its spectral radius. This proves the first inequality. The second inequality follows because the trace of $\boldsymbol{\Lambda} \boldsymbol{\Sigma}$ is no larger than $\rho(\boldsymbol{\Sigma}) \operatorname{trace}(\boldsymbol{\Lambda})=\rho(\boldsymbol{\Sigma})$.

The formulas for the worst-case and average-case errors are quite similar, and in fact, in a certain sense equivalent. This equivalence in a general setting is wellknown (e.g., see [Rit00]). It is now applied to the problem at hand.

Lemma 2. Consider the case of random quadrature rules applied to integrands that take the form of wavelet series. Let $\mathcal{H}_{\text {wav }}$ be the space defined in (12) in terms of the parameters $\omega_{u \kappa}$, and let $\tilde{\mathcal{H}}_{\text {wav }}$ be another such space defined in terms of the parameters $\tilde{\omega}_{u \kappa}=\omega_{u \kappa} \sigma_{u \kappa}$, where the $\sigma_{u \kappa}$ are the parameters used to define $\boldsymbol{\Sigma}$ in (15). For any kind of randomized quadrature rule $\mathcal{Q}_{n}$ it follows that

$$
e^{\mathrm{w}}\left(\tilde{\mathcal{H}}_{\text {wav }} ; \mathcal{Q}_{n}\right)=e^{\mathrm{a}}\left(\mathcal{H}_{\text {wav }} ; \mathcal{Q}_{n}\right)
$$

provided (17) holds.

Proof. Given the vector of wavelets $\boldsymbol{\psi}_{\omega}$ that defines the space $\mathcal{H}_{\text {wav }}$, and the vector $\boldsymbol{\psi}_{\tilde{\omega}}$ that defines the space $\tilde{\mathcal{H}}_{\text {wav }}$, it follows that $\psi_{u \kappa \tau \gamma}^{\tilde{\omega}}=\sigma_{u \kappa}^{1 / 2} \psi_{u \kappa \tau \gamma}^{\omega}$. Note that $\boldsymbol{\Sigma}$ may be written as $\boldsymbol{\Sigma}=\boldsymbol{\Sigma}^{1 / 2} \boldsymbol{\Sigma}^{1 / 2}$, where $\boldsymbol{\Sigma}^{1 / 2}$ is block diagonal with blocks $\boldsymbol{\Sigma}_{u \boldsymbol{}}^{1 / 2}=\sigma_{u \boldsymbol{\kappa}}^{1 / 2}\left[\otimes^{|u|} \mathbf{H}_{b}\right]$. By condition (6) it follows that $\left[\otimes^{|u|} \mathbf{H}_{b}\right]$, acting on the $u \boldsymbol{\kappa} \boldsymbol{\tau}$ block of $\boldsymbol{\psi}_{\omega}$, leaves it unchanged. Thus, $\boldsymbol{\psi}_{\tilde{\omega}}=\boldsymbol{\Sigma}^{1 / 2} \boldsymbol{\psi}_{\omega}$.

Let $\boldsymbol{\Lambda}$ be the matrix associated with $\mathcal{H}_{\text {wav }}$ and $\tilde{\Lambda}$ the matrix associated with $\tilde{\mathcal{H}}_{\text {wav }}$. By the definition of these matrices, Theorem 1 , and the discussion above,

$$
\begin{aligned}
\tilde{\boldsymbol{\Lambda}} & =E_{Q \in \mathcal{Q}_{n}}\left[\operatorname{Err}\left(\boldsymbol{\psi}_{\tilde{\omega}} ; Q\right) \operatorname{Err}\left(\boldsymbol{\psi}_{\tilde{\omega}} ; Q\right)^{T}\right] \\
& =E_{Q \in \mathcal{Q}_{n}}\left[\boldsymbol{\Sigma}^{1 / 2} \operatorname{Err}\left(\boldsymbol{\psi}_{\omega} ; Q\right) \operatorname{Err}\left(\boldsymbol{\psi}_{\omega} ; Q\right)^{T} \boldsymbol{\Sigma}^{1 / 2}\right]=\boldsymbol{\Sigma}^{1 / 2} \boldsymbol{\Lambda} \boldsymbol{\Sigma}^{1 / 2}
\end{aligned}
$$

It then follows that

$$
\begin{aligned}
e^{\mathrm{w}}\left(\tilde{\mathcal{H}}_{\text {wav }} ; \mathcal{Q}_{n}\right) & =\sqrt{\operatorname{trace}(\tilde{\boldsymbol{\Lambda}})}=\sqrt{\operatorname{trace}\left(\boldsymbol{\Sigma}^{1 / 2} \boldsymbol{\Lambda} \boldsymbol{\Sigma}^{1 / 2}\right)}=\sqrt{\operatorname{trace}(\boldsymbol{\Lambda} \boldsymbol{\Sigma})} \\
& =e^{\mathrm{a}}\left(\mathcal{H}_{\text {wav }} ; \mathcal{Q}_{n}\right) .
\end{aligned}
$$


Now we consider the specific randomization where the quadrature rules are scrambled as proposed by Owen. For details of this scrambling, readers are referred to [we95, Owe00] and the references therein. Although this scrambling is typically applied to $(t, m, s)$-nets and $(t, s)$-sequences, we do not yet need to assume anything about the points being scrambled. Given any $P=\left\{\mathbf{z}_{1}, \cdots, \mathbf{z}_{n}\right\}$ as the initial point set (before scrambling) to be used for quasi-Monte Carlo quadrature as defined in (1), with $w_{i}=1 / n$, we denote by $\mathcal{Q}_{\mathrm{sc}}(P)$ the space of scrambled quadratures.

Lemma 3. Let $P=\left\{\mathbf{z}_{1}, \cdots, \mathbf{z}_{n}\right\}$. Then $\boldsymbol{\Lambda}^{\mathrm{sc}}$, the matrix $\boldsymbol{\Lambda}$ defined in (13) for scrambled quadrature, is a block diagonal matrix whose $b^{|u|} \times b^{|u|}$ blocks $\Lambda_{u \kappa \tau}^{\mathrm{sc}}$ are of the form

$$
\boldsymbol{\Lambda}_{u \kappa \boldsymbol{\tau}}^{\mathrm{sc}}=n^{-1} \omega_{u \kappa} \Gamma_{u \boldsymbol{\kappa}}(P)\left[\otimes^{|u|} \mathbf{H}_{b}\right]
$$

The gain coefficients $\Gamma_{u \kappa}(P)$ of the set $P$ are defined in Owe97a as

$$
\Gamma_{u \kappa}(P)=\frac{1}{n(b-1)^{|u|}} \sum_{i, j=1}^{n} \prod_{r \in u}\left(b 1_{\left\lfloor b^{\kappa_{r}+1} z_{i r}\right\rfloor=\left\lfloor b^{\kappa_{r}+1} z_{j r}\right\rfloor}-1_{\left\lfloor b^{\kappa_{r}} z_{i r}\right\rfloor=\left\lfloor b^{\kappa_{r}} z_{j r}\right\rfloor}\right),
$$

and $\Gamma_{\emptyset}=0$.

Proof. Recall from (77) that $\int_{[0,1)^{s}} \psi_{u \kappa \tau \gamma}^{\omega}(\mathbf{x}) d \mathbf{x}=\omega_{\emptyset} \delta_{u \emptyset}$. Denote the entries of $\boldsymbol{\Lambda}^{\mathrm{sc}}$ by $\lambda_{u \kappa \tau} \boldsymbol{\gamma}, u^{\prime} \boldsymbol{\kappa}^{\prime} \boldsymbol{\tau}^{\prime} \boldsymbol{\gamma}^{\prime}$. Then

$$
\begin{aligned}
& \lambda_{u \boldsymbol{\kappa} \boldsymbol{\tau} \boldsymbol{\gamma}, u^{\prime} \boldsymbol{\kappa}^{\prime} \boldsymbol{\tau}^{\prime} \boldsymbol{\gamma}^{\prime}} \\
& \quad=n^{-2} \sum_{i, j=1}^{n} E_{Q \in \mathcal{Q}_{\mathrm{sc}}(P)}\left\{\left[\omega_{\emptyset} \delta_{u \emptyset}-\psi_{u \boldsymbol{\kappa} \boldsymbol{\tau} \boldsymbol{\gamma}}^{\omega}\left(\mathbf{x}_{i}\right)\right]\left[\omega_{\emptyset} \delta_{u^{\prime} \emptyset}-\psi_{u^{\prime} \boldsymbol{\kappa}^{\prime} \boldsymbol{\tau}^{\prime} \boldsymbol{\gamma}^{\prime}}^{\omega}\left(\mathbf{x}_{j}\right)\right]\right\} \\
& =n^{-2}\left(\omega_{u \boldsymbol{\kappa}} \omega_{u^{\prime} \boldsymbol{\kappa}^{\prime}}\right)^{1 / 2} \sum_{i, j=1}^{n} E_{Q \in \mathcal{Q}_{\mathrm{sc}}(P)}\left\{\left[\delta_{u \emptyset}-\psi_{u \boldsymbol{\kappa} \boldsymbol{\tau} \boldsymbol{\gamma}}\left(\mathbf{x}_{i}\right)\right]\left[\delta_{u^{\prime} \emptyset}-\psi_{u^{\prime} \boldsymbol{\kappa}^{\prime} \boldsymbol{\tau}^{\prime} \boldsymbol{\gamma}^{\prime}}\left(\mathbf{x}_{j}\right)\right]\right\} .
\end{aligned}
$$

From [Owe97a Lemmas 4-5] it is known that

$$
E_{Q \in \mathcal{Q}_{\mathrm{sc}}(P)}\left[\psi_{u \boldsymbol{\kappa} \boldsymbol{\gamma} \gamma}\left(\mathbf{x}_{i}\right) \psi_{u^{\prime} \boldsymbol{\kappa}^{\prime} \boldsymbol{\tau}^{\prime} \boldsymbol{\gamma}^{\prime}}\left(\mathbf{x}_{j}\right)\right]=\delta_{u u^{\prime}} \delta_{\boldsymbol{\kappa} \boldsymbol{\kappa}^{\prime}} \delta_{\boldsymbol{\tau} \boldsymbol{\tau}^{\prime}} \Upsilon_{i j u \boldsymbol{\kappa}} \prod_{r \in u}\left(\delta_{\gamma_{r} \gamma_{r}^{\prime}}-b^{-1}\right)
$$

where

$$
\Upsilon_{i j u \kappa}=\frac{1}{(b-1)^{|u|}} \prod_{r \in u}\left(b 1_{\left\lfloor b^{\kappa_{r}+1} z_{i r}\right\rfloor=\left\lfloor b^{\kappa_{r}+1} z_{j r}\right\rfloor}-1_{\left\lfloor b^{\kappa_{r}} z_{i r}\right\rfloor=\left\lfloor b^{\kappa_{r}} z_{j r}\right\rfloor}\right) .
$$

It then follows from the definition of the gain coefficients in 22 that

$$
\begin{aligned}
& \lambda_{u \boldsymbol{\kappa} \boldsymbol{\tau} \boldsymbol{\gamma}, u^{\prime} \boldsymbol{\kappa}^{\prime} \boldsymbol{\tau}^{\prime} \boldsymbol{\gamma}^{\prime}} \\
& \quad= \begin{cases}0, & \text { if } u \neq u^{\prime} \text { or } \boldsymbol{\kappa} \neq \boldsymbol{\kappa}^{\prime} \text { or } \boldsymbol{\tau} \neq \boldsymbol{\tau}^{\prime}, \\
n^{-1} \omega_{u \boldsymbol{\kappa}} \Gamma_{u \kappa}(P) \prod_{r \in u}\left(\delta_{\gamma_{r} \gamma_{r}^{\prime}}-b^{-1}\right), & \text { otherwise. }\end{cases}
\end{aligned}
$$

This completes the proof of (21). 
Given the formula for $\boldsymbol{\Lambda}^{\mathrm{sc}}$, one can now apply Theorem 1 to compute the three error criteria for scrambled quadrature rules. These calculations are feasible because $\boldsymbol{\Lambda}^{\mathrm{sc}}$ is block diagonal. The simple structure of $\boldsymbol{\Lambda}^{\mathrm{sc}}$ results from the good match between the space of integrands $\mathcal{H}_{\text {wav }}$ and Owen's scrambled quadrature rules $\mathcal{Q}_{\text {sc }}(P)$.

Theorem 4. Consider the case of Owen's scrambled quadrature rules applied to integrands that take the form of wavelet series. The three error criteria defined in (2) are given by

$$
\begin{aligned}
& e^{\mathrm{w}}\left(\mathcal{H}_{\mathrm{wav}} ; \mathcal{Q}_{\mathrm{sc}}(P)\right)=n^{-1 / 2}\left[\sum_{u \neq \emptyset, \kappa}(b-1)^{|u|} b^{|\kappa|} \omega_{u \kappa} \Gamma_{u \kappa}(P)\right]^{1 / 2}, \\
& \text { assuming (14), } \\
& e^{\mathrm{r}}\left(\mathcal{H}_{\text {wav }} ; \mathcal{Q}_{\mathrm{sc}}(P)\right)=n^{-1 / 2}\left[\max _{u \neq \emptyset, \kappa}\left\{\omega_{u \kappa} \Gamma_{u \kappa}(P)\right\}\right]^{1 / 2} \text {, } \\
& e^{\mathrm{a}}\left(\mathcal{H}_{\mathrm{wav}} ; \mathcal{Q}_{\mathrm{sc}}(P)\right)=n^{-1 / 2}\left[\sum_{u \neq \emptyset, \kappa}(b-1)^{|u|} b^{|\kappa|} \sigma_{u \kappa} \omega_{u \kappa} \Gamma_{u \kappa}(P)\right]^{1 / 2}, \\
& \text { assuming (17). }
\end{aligned}
$$

Proof. To prove (23a), note from (18a) that $e^{\mathrm{w}}\left(\mathcal{H}_{\text {wav }} ; \mathcal{Q}_{\mathrm{sc}}(P)\right)=\sqrt{\operatorname{trace}\left(\boldsymbol{\Lambda}^{\mathrm{sc}}\right)}$. This trace can then be computed by Lemma 3 in straightforward manner to give (23a):

$$
\operatorname{trace}\left(\boldsymbol{\Lambda}^{\mathrm{sc}}\right)=\sum_{u \neq \emptyset, \kappa, \tau} \operatorname{trace}\left(\boldsymbol{\Lambda}_{u \kappa \boldsymbol{\tau}}^{\mathrm{sc}}\right)=\sum_{u \neq \emptyset, \kappa} n^{-1}(b-1)^{|u|} b^{|\kappa|} \omega_{u \kappa} \Gamma_{u \kappa} .
$$

Formula 23b) follows directly from (18b) and Lemma 3 Formula (23c) is a consequence of (23a) and Lemma 2

\section{AsYmptotic BeHAVIOR OF ERROR CRITERIA FOR NETS}

The gain coefficients were defined to measure the performance of an arbitrary set against a simple random sample. Let $\mathcal{Q}_{\mathrm{mc}, n}$ denote the sample space of the simple Monte Carlo quadrature, that is, the points in $P \in \mathcal{Q}_{\mathrm{mc}, n}$ are independent and uniformly distributed over $[0,1)^{s}$ random variables and the weights are $w_{i}=1 / n$. The space $\mathcal{Q}_{\mathrm{mc}, n}$ is invariant under scrambling. Moreover, $E_{P \in \mathcal{Q}_{\mathrm{mc}, n}} \Gamma_{u \kappa}(P)=1$ for all $u \kappa$. This leads to the following formulas in (24) for the error criteria for classical Monte Carlo quadrature. Note that these three error criteria all decay proportionally to $n^{-1 / 2}$, but the constants in front are largest for the worst-case and smallest for the average-case.

Corollary 5. Consider the case of Monte Carlo quadrature rules based on simple random samples applied to integrands that take the form of wavelet series. The 
three error criteria defined in (2) are

$$
\begin{aligned}
& e^{\mathrm{w}}\left(\mathcal{H}_{\mathrm{wav}} ; \mathcal{Q}_{\mathrm{mc}, n}\right)=n^{-1 / 2}\left[\sum_{u \neq \emptyset, \kappa}(b-1)^{|u|} b^{|\kappa|} \omega_{u \kappa}\right]^{1 / 2}, \text { assuming (14) } \\
& e^{\mathrm{r}}\left(\mathcal{H}_{\text {wav }} ; \mathcal{Q}_{\mathrm{mc}, n}\right)=n^{-1 / 2}\left[\max _{u \neq \emptyset, \kappa}\left\{\omega_{u \kappa}\right\}\right]^{1 / 2}, \\
& e^{\mathrm{a}}\left(\mathcal{H}_{\text {wav }} ; \mathcal{Q}_{\mathrm{mc}, n}\right)=n^{-1 / 2}\left[\sum_{u \neq \emptyset, \kappa}(b-1)^{|u|} b^{|\kappa|} \sigma_{u \kappa} \omega_{u \kappa}\right]^{1 / 2},
\end{aligned}
$$

In order for the error criteria to decay faster than $\mathrm{O}\left(n^{-1 / 2}\right)$, the gain coefficients must become small or even zero, especially for larger $\omega_{u \kappa}$. The gain coefficients for $(\lambda, 0, m, s)$-nets and $(0, s)$-sequences in base $b$ can be computed precisely Owe97a. HY00. For general $(\lambda, t, m, s)$-nets and $(t, s)$-sequences with $t>0$, one can only obtain upper bounds on the gain coefficients Owe97a, HY00. In the case of digital nets and sequences, precise formulae for the gain coefficients can be obtained NP01. YH02, but these offer no improvement in the asymptotic decay rates over the upper bounds on the gain coefficients for general nets and sequences. The results mentioned in this paragraph are summarized in the following two lemmas.

Lemma 6. Let $P$ be $a(\lambda, t, m, s)$-net in base $b$. Then the gain coefficients of $P$ have the following upper bounds:

$$
\begin{aligned}
\Gamma_{u \kappa}(P) & =0, & |\boldsymbol{\kappa}| \leq m-t-|u|, \\
\Gamma_{u \boldsymbol{\kappa}}(P) & \leq b^{t}\left[\frac{b+1}{b-1}\right]^{|u|}, & m-t-|u|<|\boldsymbol{\kappa}| \leq m-t, \\
\Gamma_{u \kappa}(P) & \leq b^{t}\left[\frac{b^{|u|}+(b-2)^{|u|}}{2(b-1)^{|u|}}\right], & |\boldsymbol{\kappa}|>m-t .
\end{aligned}
$$

For the case of $t=0$, the gain coefficients can be computed exactly as

$$
\Gamma_{u \kappa}(P)=\Gamma_{u \kappa}^{0-\text { net }}(\lambda, m)=\frac{1}{(b-1)^{|u|}} \sum_{\alpha=1}^{-m+|\kappa|+|u|-1}\left(\begin{array}{c}
|u| \\
\alpha
\end{array}\right)(-1)^{\alpha}\left[b^{|u|-\alpha}-\lambda b^{m-|\kappa|}\right] .
$$

The right-hand side reduces to 0 for $|\boldsymbol{\kappa}| \leq m-|u|$ and 1 for $|\boldsymbol{\kappa}| \geq m+1$. Let $P$ contain the first $n$ terms of a $(t, s)$-sequence in base $b$. Let $n$ be expressed as

$$
n=\lambda_{0}+\lambda_{1} b+\cdots+\lambda_{q} b^{q}=\cdots 0 \lambda_{q} \lambda_{q-1} \cdots \lambda_{1} \lambda_{0} \text { (base } b \text { ), }
$$

where $q=\left\lfloor\log _{b}(n)\right\rfloor$ and where $\lambda_{m} \in\{0,1, \ldots, b-1\}$. Then the gain coefficients of $P$ have the following upper bounds:

$$
\Gamma_{u \kappa}(P) \leq \frac{1}{n} b^{t} b^{\min (t+|\kappa|+|u|, q+1)}\left(\frac{b+1}{b-1}\right)^{|u|+1} \leq b^{t+1}\left(\frac{b+1}{b-1}\right)^{|u|+1} .
$$

For $t=0$, these gain coefficients can be computed precisely as

$$
\Gamma_{u \kappa}(P)=\frac{1}{n b(b-1)} \sum_{m=0}^{q+1} b^{m} C_{m} \Gamma_{u \kappa}^{0-\text { net }}(1, m),
$$


where $C_{0}=b \lambda_{0}\left(b-\lambda_{0}\right)$,

$$
\begin{aligned}
C_{m}= & \lambda_{m-1}\left(\lambda_{m-1}-1\right)+b \lambda_{m}\left(b-\lambda_{m}\right)-2 \lambda_{m-1} \lambda_{m} \\
& +2\left(\lambda_{m-1}-\lambda_{m}\right) b^{-m+1} \sum_{h=0}^{m-2} \lambda_{h} b^{h}, \quad m=1, \ldots, q,
\end{aligned}
$$

and

$$
C_{q+1}=\lambda_{q}\left(\lambda_{q}-1\right)+2 \lambda_{q} b^{-q} \sum_{h=0}^{q-1} \lambda_{h} b^{h} .
$$

Lemma 7. Let $a>0$ be a scalar, and let $u$ be a subset of $\{1, \ldots, s\}$. If $P$ is a $(\lambda, t, m, s)$-net in base $b$, then

$$
n^{-1} \sum_{\kappa} b^{-a|\kappa|} \Gamma_{u \kappa}(P)=O\left(n^{-1-a}[\log n]^{s-1}\right),
$$

where $n=\lambda b^{m}$. If $P$ consists of the first $n$ points of a $(t, s)$-sequence in base $b$, then

$$
n^{-1} \sum_{\kappa} b^{-a|\kappa|} \Gamma_{u \kappa}(P)= \begin{cases}O\left(n^{-1-a}[\log n]^{s-1}\right), & 0<a<1, \\ O\left(n^{-2}[\log n]^{s}\right), & a=1, \\ O\left(n^{-2}[\log n]^{s-1}\right), & a>1,\end{cases}
$$

with constants in the $O$-notation not depending on $n$ and $P$.

According to Theorem 11 the asymptotic convergence rates of the three error criteria depend not only on the decay rates of the gain coefficients, which are given in the lemma above, but also on the decay rates of the $\omega_{u \kappa}$ (and $\sigma_{u \kappa}$ for the average case). From here on it is assumed that

$$
\omega_{u \kappa}=\theta_{0} \theta_{1}^{|u|} b^{-2 \alpha|\kappa|}, \quad \sigma_{u \kappa}=\left\{1+\left[\frac{\eta(b-1)}{1-b^{1-2 \beta}}\right]\right\}^{-s} \eta^{|u|} b^{-2 \beta|\kappa|},
$$

where $\theta_{0}, \theta_{1}, \eta$ are positive numbers, and $\alpha$ is nonnegative. The summability condition in (14) for worst-case analysis is equivalent to $\alpha>1 / 2$, while condition (11) is equivalent to $\alpha \geq 0$. Condition (17) for the average-case analysis is equivalent to $\beta>1 / 2$. The constants $\alpha$ and $\beta$ determine the asymptotic rates of decay of the three error criteria.

Let $\mathcal{Q}_{\mathrm{sc}, n}$ denote Owen's scrambled quasi-Monte Carlo quadrature using a $(\lambda, t, m, s)$-net with $n=\lambda b^{m}$ points as the node set, where $\lambda=0,1, \ldots, b-1$ and $m=0,1, \ldots$.

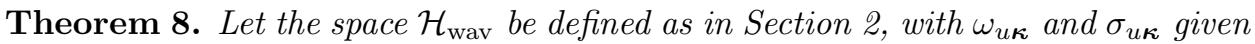
as in (25). Then for scrambled $(\lambda, t, m, s)$-nets in base $b$ the three error criteria have the following asymptotic orders:

$$
\begin{aligned}
e^{\mathrm{w}}\left(\mathcal{H}_{\mathrm{wav}} ; \mathcal{Q}_{\mathrm{sc}, n}\right) & =\mathrm{O}\left(n^{-\alpha}[\log n]^{(s-1) / 2}\right), \quad \alpha>1 / 2, \\
e^{\mathrm{r}}\left(\mathcal{H}_{\mathrm{wav}} ; \mathcal{Q}_{\mathrm{sc}, n}\right) & =\mathrm{O}\left(n^{-\alpha-1 / 2}\right), \quad \alpha \geq 0, \\
e^{\mathrm{a}}\left(\mathcal{H}_{\mathrm{wav}} ; \mathcal{Q}_{\mathrm{sc}, n}\right) & =\mathrm{O}\left(n^{-\alpha-\beta}[\log n]^{(s-1) / 2}\right), \quad \alpha \geq 0, \beta>1 / 2 .
\end{aligned}
$$

Proof. This theorem is obtained by applying the formulas in Lemma 7 to those of Theorem 11 under the assumption of (25). The key step is to note that the value of $a$ in the term $b^{-a|\kappa|}$ determines the asymptotic rate of decay. 

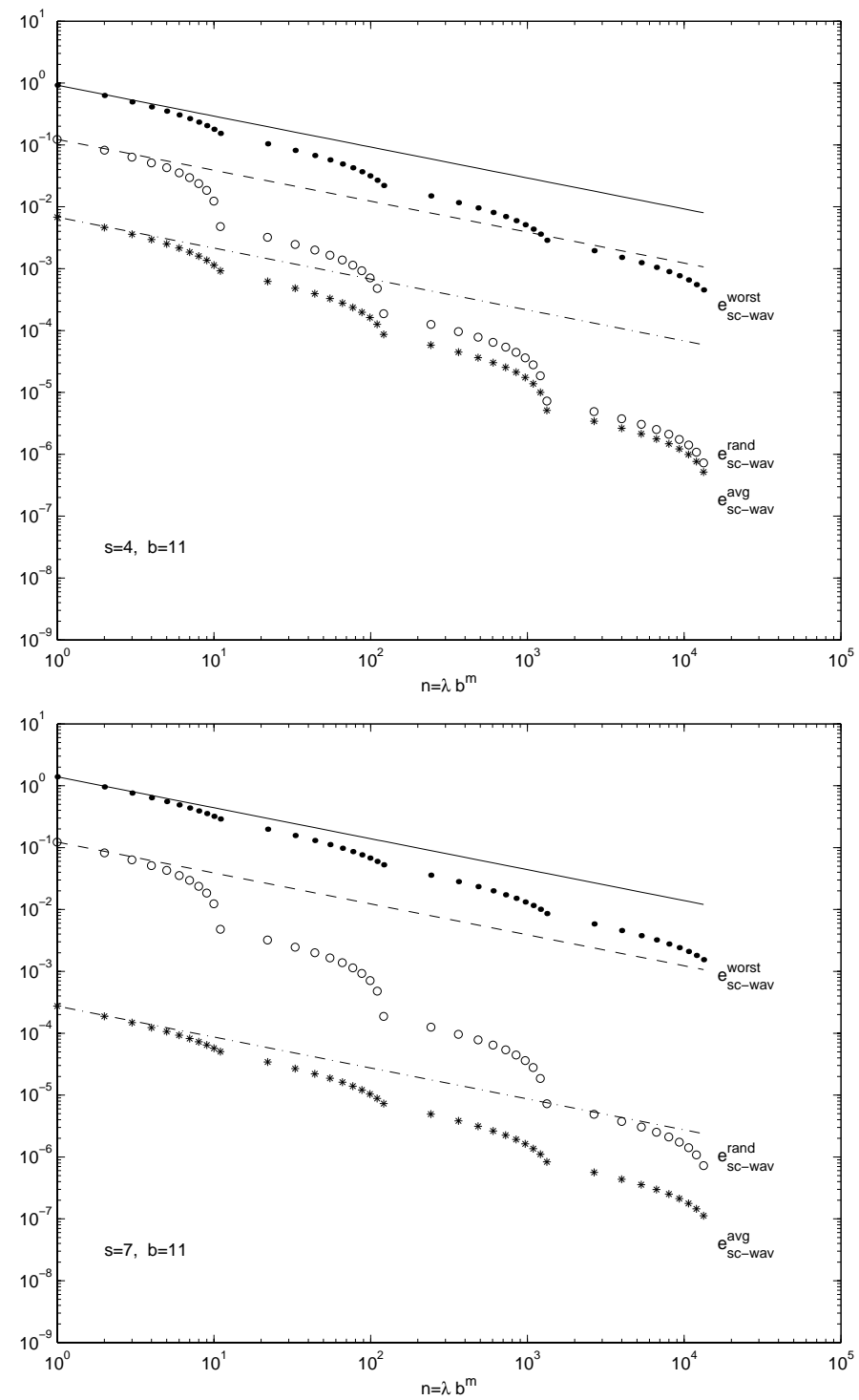

Figure 1. Performance of scrambled $(\lambda, 0, m, s)$-net quadrature for the space $\mathcal{H}_{\text {wav }}$ with the $\omega_{u \kappa}$ and $\sigma_{u \kappa}$ specified in (27). The three reference lines are for Monte Carlo quadrature: $e^{\mathrm{w}}\left(\mathcal{H}_{\mathrm{wav}} ; \mathcal{Q}_{\mathrm{mc}, n}\right) \quad($ solid $), e^{\mathrm{r}}\left(\mathcal{H}_{\mathrm{wav}} ; \mathcal{Q}_{\mathrm{mc}, n}\right)$ (dashed) and $e^{\mathrm{a}}\left(\mathcal{H}_{\text {wav }} ; \mathcal{Q}_{\mathrm{mc}, n}\right)$ (dot-dashed), respectively.

In the worst-case and average-case settings there are factors of $\log n$, but these factors disappear in the random-case. The reason for this is the structure of $\boldsymbol{\Lambda}^{\mathrm{sc}}$. In the random-case setting one looks for the maximum eigenvalue of $\boldsymbol{\Lambda}^{\mathrm{sc}}$, but in the worst-case and average-case settings one must sum up on the order of $[\log n]^{s-1}$ eigenvalues of the same size. 
The formulae given in Theorem 4 have been evaluated numerically for scrambled $(\lambda, 0, m, s)$-nets in base 11 with $\lambda=1, \ldots, 10$ and for $s=4,7$. The $\omega_{u \kappa}$ and $\sigma_{u \kappa}$ are assumed to be as follows:

$$
\omega_{u \kappa}=(6 b)^{-|u|} b^{-2|\kappa|}, \quad \sigma_{u \kappa}=\left(b^{s}-1\right)^{-1}\left(1-b^{-1}\right)^{|u|} b^{-2|\kappa|} .
$$

This corresponds to a choice of $\alpha=\beta=1$ in (25), with the values of $\theta_{0}, \theta_{1}$, and $\eta$ being chosen for convenience. Conditions (14) and (17) are automatically satisfied. The worst-case, average-case, and random-case errors for these scrambled nets are shown in Figure 1. These plots obey the inequalities $e^{\mathrm{w}}\left(\mathcal{H}_{\mathrm{wav}} ; \mathcal{Q}_{\mathrm{sc}, n}\right) \geq$ $e^{\mathrm{r}}\left(\mathcal{H}_{\text {wav }} ; \mathcal{Q}_{\mathrm{sc}, n}\right) \geq e^{\mathrm{a}}\left(\mathcal{H}_{\text {wav }} ; \mathcal{Q}_{\mathrm{sc}, n}\right)$ given in $(20)$. The three error criteria for simple Monte Carlo quadrature as given in Theorem 5 are also shown for comparison.

The Monte Carlo error criteria all decay proportional to $n^{-1 / 2}$. These lines coincide with the scrambled nets for $n=1$, and the error criterion for scrambled net quadrature is never larger than the corresponding criterion for simple Monte Carlo. The random-case error criterion for scrambled nets decays like $\mathrm{O}\left(n^{-3 / 2}\right)$, as expected. Apart from the $[\log n]^{(s-1) / 2}$ terms, worst-case and average-case criteria for nets should decay like $\mathrm{O}\left(n^{-1}\right)$ and $\mathrm{O}\left(n^{-2}\right)$, respectively. Their slower than expected decay is due to the powers of $\log n$. However, since $e^{\mathrm{r}}\left(\mathcal{H}_{\text {wav }} ; \mathcal{Q}_{\mathrm{sc}, n}\right) \geq$ $e^{\mathrm{a}}\left(\mathcal{H}_{\text {wav }} ; \mathcal{Q}_{\mathrm{sc}, n}\right)$, the powers of $\log n$ can never make the average-case error worse than the random-case. As the dimension $s$ increases, $e^{\mathrm{w}}\left(\mathcal{H}_{\text {wav }} ; \mathcal{Q}_{\mathrm{sc}, n}\right)$ increases, $e^{\mathrm{r}}\left(\mathcal{H}_{\text {wav }} ; \mathcal{Q}_{\mathrm{sc}, n}\right)$ stays about the same, and $e^{\mathrm{a}}\left(\mathcal{H}_{\text {wav }} ; \mathcal{Q}_{\mathrm{sc}, n}\right)$ decreases.

\section{LOWER BOUNDS FOR THE ERROR CRITERIA}

In the previous section the asymptotic convergence rates of worst-case, randomcase and average-case quadrature errors were determined for scrambled net quadrature applied to integrands in $\mathcal{H}_{\text {wav }}$. In this section it is shown that these convergence rates are optimal, i.e., they are the best possible for any random quadrature rule.

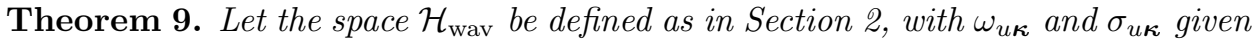
as in (25). Then the worst-case and average-case error criteria for this space as defined in (3) have the lower bounds

$$
\begin{gathered}
e^{\mathrm{w}}\left(\mathcal{H}_{\mathrm{wav}}, n\right) \geq C_{\mathrm{w}} n^{-\alpha}[\log n]^{(s-1) / 2}, \quad \alpha>1 / 2, \\
e^{\mathrm{a}}\left(\mathcal{H}_{\mathrm{wav}}, n\right) \geq C_{\mathrm{a}} n^{-\alpha-\beta}[\log n]^{(s-1) / 2}, \quad \alpha \geq 0, \beta>1 / 2,
\end{gathered}
$$

for some constants $C_{\mathrm{w}}$ and $C_{\mathrm{a}}$ that are independent of $n$.

Proof. This proof proceeds by constructing a function in $\mathcal{H}_{\text {wav }}$ that cannot be integrated very well by any quadrature rule. Let $n$ be any given positive integer. Choose an integer $m$ such that

$$
b^{m-1}<2 n \leq b^{m} .
$$

For each $s$-vector $\boldsymbol{\nu}=\left(\nu_{1}, \ldots, \nu_{s}\right)$ of nonnegative integers and each $s$-vector $\boldsymbol{\ell}=$ $\left(\ell_{1}, \ldots, \ell_{s}\right)$ of integers $0 \leq \ell_{r}<b^{\nu_{j}}$, define the elementary interval in base $b$ as

$$
B_{\boldsymbol{\nu} \ell}=\prod_{r=1}^{s}\left[\frac{\ell_{r}}{b^{\nu_{r}}}, \frac{\ell_{r}+1}{b^{\nu_{r}}}\right) .
$$

It is clear that the set of all $B_{\boldsymbol{\nu} \boldsymbol{\ell}}$ is a partition of $[0,1)^{s}$ into $b^{|\boldsymbol{\nu}|}$ elementary intervals of volume $b^{-|\boldsymbol{\nu}|}$. 
Let $P=\left\{\mathbf{x}_{1}, \ldots, \mathbf{x}_{n}\right\} \subset[0,1)^{s}$ be any given set of points. We define the function $f_{\boldsymbol{\nu}}$ for each $\boldsymbol{\nu}$ with $|\boldsymbol{\nu}|=m$ by

$$
f_{\boldsymbol{\nu}}(\mathbf{x})= \begin{cases}1, & \text { for all } \mathbf{x} \in B_{\boldsymbol{\nu} \ell} \text { with } B_{\boldsymbol{\nu} \ell} \cap P=\emptyset \\ 0, & \text { otherwise. }\end{cases}
$$

Then $f_{\nu} \in \mathcal{H}_{\text {wav }}$ and

$$
I\left(f_{\boldsymbol{\nu}}\right)=\int_{[0,1)^{s}} f_{\boldsymbol{\nu}}(\mathbf{x}) d \mathbf{x} \geq b^{-m}\left(b^{m}-n\right) \geq \frac{1}{2},
$$

since $2 n \leq b^{m}$ from (30). Now we define $f_{0}=\sum_{|\boldsymbol{\nu}|=m} f_{\boldsymbol{\nu}}$. Then $f_{0} \in \mathcal{H}_{\text {wav }}$, $f_{0}\left(\mathbf{x}_{i}\right)=0$ for $i=1, \ldots, n$, and

$$
\operatorname{Err}\left(f_{0}, Q\right)=I\left(f_{0}\right)=\sum_{|\boldsymbol{\nu}|=m} I\left(f_{\boldsymbol{\nu}}\right) \geq \frac{1}{2}\left(\begin{array}{c}
m+s-1 \\
s-1
\end{array}\right) \geq c_{1} m^{s-1}
$$

where $c_{1}$ is a positive constant.

To complete the proof, the norm of $f_{0}$ must be estimated. For any $|u|$-vector $\kappa$ and any $s$-vector $\boldsymbol{\nu}$, the notation $\boldsymbol{\kappa} \leq \boldsymbol{\nu}$ means that $\kappa_{r} \leq \nu_{r}$ for all $r \in u$. The wavelet series expansion for the function $f_{\nu}$ defined above is a finite sum:

$$
f_{\boldsymbol{\nu}}(\mathbf{x})=\sum_{\boldsymbol{\kappa} \leq \boldsymbol{\nu}, \boldsymbol{\tau} \boldsymbol{\gamma}} \hat{f}_{\boldsymbol{\nu}, u \kappa \boldsymbol{\tau} \boldsymbol{\gamma}} \psi_{u \kappa \boldsymbol{\tau} \boldsymbol{\gamma}}(\mathbf{x}), \quad \text { where } \hat{f}_{\boldsymbol{\nu}, u \kappa \tau \boldsymbol{\gamma}}=\int_{[0,1)^{s}} f_{\boldsymbol{\nu}}(\mathbf{x}) \psi_{u \kappa \boldsymbol{\tau} \boldsymbol{\gamma}}(\mathbf{x}) d \mathbf{x}
$$

Note that the set of support for $\psi_{u \boldsymbol{\kappa} \boldsymbol{\tau} \boldsymbol{\gamma}}$ is $B_{(\boldsymbol{\kappa}, \mathbf{0}) \boldsymbol{\tau}}$ by the definition (31), where $(\boldsymbol{\kappa}, \mathbf{0})$ is the $s$-vector obtained by padding $\boldsymbol{\kappa}$ with zeros for all $r \notin u$. Since $\left\|f_{\nu}\right\|_{\infty}=1$ and $\left\|\psi_{u \kappa \tau \gamma}\right\|_{\infty}=b^{(|\kappa|-|u|) / 2}(b-1)^{|u|}$, it follows that

$$
\begin{aligned}
\left|\hat{f}_{\boldsymbol{\nu}, u \boldsymbol{\kappa} \boldsymbol{\gamma} \gamma}\right| & =\left|\int_{B_{(\boldsymbol{\kappa}, \mathbf{0}) \boldsymbol{\tau}}} f_{\boldsymbol{\nu}}(\mathbf{x}) \psi_{u \boldsymbol{\tau} \boldsymbol{\gamma} \boldsymbol{\gamma}}(\mathbf{x}) d \mathbf{x}\right| \\
& \leq b^{-|\boldsymbol{\kappa}|} b^{(|\boldsymbol{\kappa}|-|u|) / 2}(b-1)^{|u|}=(b-1)^{|u|} b^{-(|\boldsymbol{\kappa}|+|u|) / 2} .
\end{aligned}
$$

Based on the above upper bound, the square norm of $f_{0}$ can now be written as follows:

$$
\begin{aligned}
& \left\|f_{0}\right\|_{\mathcal{H}_{\text {wav }}}^{2}=\left\langle f_{0}, f_{0}\right\rangle_{\mathcal{H}_{\text {wav }}}=\sum_{|\boldsymbol{\nu}|=\left|\boldsymbol{\nu}^{\prime}\right|=m}\left\langle f_{\boldsymbol{\nu}}, f_{\boldsymbol{\nu}^{\prime}}\right\rangle_{\mathcal{H}_{\mathrm{wav}}} \\
& \leq \sum_{|\boldsymbol{\nu}|=\left|\boldsymbol{\nu}^{\prime}\right|=m} \sum_{\substack{u \subseteq\{1, \ldots, s\} \\
\boldsymbol{\kappa} \leq \min \left(\boldsymbol{\nu}, \boldsymbol{\nu}^{\prime}\right)}} \sum_{\boldsymbol{\tau} \boldsymbol{\gamma}} \omega_{u \kappa}^{-1}\left|\hat{f}_{\boldsymbol{\nu}, u \kappa \tau \gamma}\right|\left|\hat{f}_{\boldsymbol{\nu}^{\prime}, u \kappa \tau \boldsymbol{\gamma}}\right| \\
& \leq \sum_{\substack{u \subseteq\{1, \ldots, s\} \\
0 \leq|\boldsymbol{\kappa}| \leq m}} \sum_{\substack{\boldsymbol{\nu}, \boldsymbol{\nu}^{\prime} \geq \boldsymbol{\kappa} \\
|\boldsymbol{\nu}|=\left|\boldsymbol{\nu}^{\prime}\right|=m}} \theta_{0}^{-1} \theta_{1}^{-|u|} b^{2 \alpha|\boldsymbol{\kappa}|}(b-1)^{2|u|} \\
& \leq \theta_{0}^{-1} \max \left(1, \theta_{1}^{-s}\right)(b-1)^{2 s} \sum_{p=0}^{m} b^{2 \alpha p}\left[\sum_{\substack{u \subseteq\{1, \ldots, s\} \\
|\boldsymbol{\kappa}|=p}} \sum_{\substack{\boldsymbol{\nu}, \boldsymbol{\nu}^{\prime} \geq \boldsymbol{\kappa} \\
|\boldsymbol{\nu}-(\boldsymbol{\kappa}, \mathbf{0})|=\left|\boldsymbol{\nu}^{\prime}-(\boldsymbol{\kappa}, \mathbf{0})\right|=m-p}} 1\right] .
\end{aligned}
$$

Given $u$ and $\kappa$, there are $\left(\begin{array}{c}m-p+s-1 \\ s-1\end{array}\right) \leq(m-p+s-1)^{s-1}$ ways to choose a nonnegative integer $s$-vector $\boldsymbol{\nu}-(\boldsymbol{\kappa}, \mathbf{0})$ with cardinality $m-p$. Moreover, there are 
$\left(\begin{array}{l}s \\ q\end{array}\right)$ subsets $u$ with $|u|=q$, and for each of them $\left(\begin{array}{c}p+q-1 \\ q-1\end{array}\right)$ ways to construct a $\boldsymbol{\kappa}$ with $|\boldsymbol{\kappa}|=p$. Since

$$
\sum_{q=0}^{s}\left(\begin{array}{l}
s \\
q
\end{array}\right)\left(\begin{array}{c}
p+q-1 \\
q-1
\end{array}\right) \leq 2^{s}(p+s-1)^{s-1} \leq 2^{s}(m+s-1)^{s-1}
$$

the upper bound on $\left\|f_{0}\right\|_{\mathcal{H}_{\text {wav }}}^{2}$ may be written as

$$
\begin{gathered}
\left\|f_{0}\right\|_{\mathcal{H}_{\text {wav }}}^{2} \leq \theta_{0}^{-1} \max \left(1, \theta_{1}^{-s}\right)(b-1)^{2 s} 2^{s}(m+s-1)^{s-1} \\
\times \sum_{p=0}^{m}(m-p+s-1)^{2(s-1)} b^{2 \alpha p} \\
\leq \theta_{0} \max \left(1, \theta_{1}^{-s}\right)(b-1)^{2 s} 2^{s}(m+s-1)^{s-1} b^{2 \alpha m} \\
\times \sum_{q=0}^{m}(q+s-1)^{2(s-1)} b^{-2 \alpha q} \\
\leq c_{2} m^{s-1} b^{2 \alpha m}
\end{gathered}
$$

for some $c_{2}$ depending on $b, s$, and $\alpha$, but not on $m$.

Letting $f_{*}=f_{0} /\left\|f_{0}\right\|_{\mathcal{H}_{\text {wav }}}$, we see that $f_{*}$ lies in the unit ball $\mathcal{B}_{\text {wav }}$. Moreover, from (301), (32) and (33) it follows that

$$
\left|\operatorname{Err}\left(f_{*}, Q\right)\right| \geq \frac{c_{1} m^{s-1}}{\sqrt{c_{2} m^{s-1} b^{2 \alpha m}}}=\frac{c_{1}}{\sqrt{c_{2}}} m^{(s-1) / 2} b^{-\alpha m} \geq C_{\mathrm{w}} n^{-\alpha}[\log n]^{(s-1) / 2}
$$

for some constant $C_{\mathrm{w}}$. This implies (28). Formula (29) then follows from Lemma 2 .

Theorem 10. Let the space $\mathcal{H}_{\mathrm{wav}}$ be defined as in Section 2 with $\omega_{u \kappa}$ and $\sigma_{u \kappa}$ given as in (25). Then the random-case error criterion for this space as defined in (3) has the lower bound

$$
e^{\mathrm{r}}\left(\mathcal{H}_{\text {wav }}, n\right) \geq C_{\mathrm{r}} n^{-\alpha-1 / 2}, \quad \alpha \geq 0,
$$

for some constant $C_{\mathrm{r}}$ that is independent of $n$.

Proof. It is sufficient to consider the case with $s=1$, since integration in the space $\mathcal{H}_{\text {wav }}$ with $s=1$ is no harder than integration in the respective $\mathcal{H}_{\text {wav }}$ with $s>1$ (the one-dimensional $\mathcal{H}_{\text {wav }}$ can be identified with the subspace of the multidimensional $\mathcal{H}_{\text {wav }}$ consisting of functions depending only on the first variable, or equivalently, of functions $f$ with $\hat{f}_{u \kappa \tau \gamma}=0$ for all $u$ except $u=\emptyset$ and $u=\{1\}$ ). By convention the weights $\omega_{u \kappa}$ in (25) for the one-dimensional case are written as $\omega_{\emptyset}=\theta_{0}$ and $\omega_{\kappa}=\theta_{0} b^{-2 \alpha \kappa}$, for $\kappa=0,1,2, \ldots$ The square norm of the function in $\mathcal{H}_{\text {wav }}$ with $s=1$ is

$$
\|f\|^{2}=\hat{f}_{\emptyset}^{2} \omega_{\emptyset}^{-1}+\sum_{\kappa=0}^{\infty} \sum_{\tau=0}^{b^{\kappa}-1} \sum_{\gamma=0}^{b-1} \hat{f}_{\kappa \tau \gamma}^{2} \omega_{\kappa}^{-1},
$$

where

$$
\hat{f}_{\emptyset}=\int_{0}^{1} f(x) d x \quad \text { and } \quad \hat{f}_{\kappa \tau \gamma}=\int_{0}^{1} f(x) \psi_{\kappa \tau \gamma}(x) d x
$$


Let $n, m$ be taken as in (30) in the proof of the previous theorem. Again define basic intervals

$$
B_{m \ell}=\left[\frac{\ell}{b^{m}}, \frac{\ell+1}{b^{m}}\right), \quad \ell=0,1, \ldots, b^{m}-1,
$$

and let $g_{\ell}=1_{B_{m \ell}}(x)$ be the indicator function of $B_{m \ell}$. It follows that $I\left(g_{\ell}\right)=b^{-m}$. Define

$$
g=\sum_{\ell=0}^{b^{m}-1} \varepsilon_{\ell} g_{\ell}, \quad \text { where } \varepsilon_{\ell} \in\{1,-1\} .
$$

The norm of this function can be bounded similarly to the proof of the previous theorem. First the series coefficients of $g$ are bounded as follows: $\left|\hat{g}_{\emptyset}\right| \leq 1$ and

$$
\left|\hat{g}_{\kappa \tau \gamma}\right|=\left|\int_{\tau b^{-\kappa}}^{(\tau+1) b^{-\kappa}} g(x) \psi_{\kappa \tau \gamma}(x) d x\right| \leq b^{(\kappa-1) / 2}(b-1) b^{-\kappa}=b^{-(\kappa+1) / 2}(b-1) .
$$

This gives an upper bound on the norm of $g$ :

$$
\|g\|_{\mathcal{H}_{\text {wav }}}^{2}=\omega_{\emptyset}^{-1}+\sum_{\kappa=0}^{m} \sum_{\tau=0}^{b^{\kappa}-1} \sum_{\gamma=0}^{b-1} \hat{g}_{\kappa \tau \gamma}^{2} \omega_{\kappa}^{-1} \leq \theta_{0}^{-1}+\theta_{0}^{-1}(b-1)^{2} \sum_{\kappa=0}^{m} b^{2 \alpha \kappa} \leq c_{3}^{2} b^{2 \alpha m},
$$

for some constant $c_{3}$ that is independent of $m$.

Now consider the functions $f_{\ell}=g_{\ell} /\left(c_{3} b^{\alpha m}\right)$. These $f_{\ell}$ have disjoint supports and satisfy

$$
I\left(f_{\ell}\right) \geq c_{3} b^{-(\alpha+1) m}, \quad\left\|\sum_{\ell=0}^{b^{m}-1} \varepsilon_{\ell} f_{\ell}\right\| \leq 1 \text { for any } \varepsilon_{\ell} \in\{1,-1\} .
$$

From [Nov88, Section 2.2.4, Proposition 1] it follows that

$$
e^{\mathrm{r}}\left(\mathcal{H}_{\text {wav }}, n\right) \geq c_{4} b^{-(\alpha+1) m}\left(b^{m}\right)^{1 / 2}=c_{4} b^{-(\alpha+1 / 2) m} \geq C_{\mathrm{r}} n^{-\alpha-1 / 2} .
$$

This completes the proof of (34).

Theorems 8 9. and 10 may be combined to summarize the main results of this article, namely that scrambled net quadrature is optimal for Haar wavelet series in all three settings. We use the standard notation $a_{n} \asymp b_{n}$ for nonnegative sequences $a_{n}$ and $b_{n}$, meaning that there are constants $c_{\mathrm{lo}}, c_{\mathrm{up}}>0$ such that $c_{\mathrm{lo}} b_{n} \leq a_{n} \leq$ $c_{\text {up }} b_{n}$.

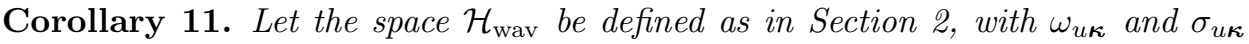
given as in (25). Let $\mathcal{Q}_{\mathrm{sc}, n}$ denote Owen's scrambled quasi-Monte Carlo quadrature using a $(\lambda, t, m, s)$-net with $\lambda b^{m}$ points as the node set, where $\lambda=0,1, \ldots, b-1$ and $m=0,1, \ldots$ Then,

$$
\begin{gathered}
\min _{\lambda b^{m} \leq n} e^{\mathrm{w}}\left(\mathcal{H}_{\mathrm{wav}} ; \mathcal{Q}_{\mathrm{sc}, \lambda b^{m}}\right) \asymp e^{\mathrm{w}}\left(\mathcal{H}_{\text {wav }}, n\right) \asymp n^{-\alpha}[\log n]^{(s-1) / 2}, \quad \alpha>1 / 2, \\
\min _{\lambda b^{m} \leq n} e^{\mathrm{r}}\left(\mathcal{H}_{\mathrm{wav}} ; \mathcal{Q}_{\mathrm{sc}, \lambda b^{m}}\right) \asymp e^{\mathrm{r}}\left(\mathcal{H}_{\text {wav }}, n\right) \asymp n^{-\alpha-1 / 2}, \quad \alpha \geq 0, \\
\min _{\lambda b^{m} \leq n} e^{\mathrm{a}}\left(\mathcal{H}_{\mathrm{wav}} ; \mathcal{Q}_{\mathrm{sc}, \lambda b^{m}}\right) \asymp e^{\mathrm{a}}\left(\mathcal{H}_{\text {wav }}, n\right) \asymp n^{-\alpha-\beta}[\log n]^{(s-1) / 2}, \quad \alpha \geq 0, \beta>1 / 2 .
\end{gathered}
$$

Remark. Usually the gap between worst-case and random-case $n$-th minimal errors is not of order more than $n^{1 / 2}$. This is the first time that the authors have encountered a situation where this gap is larger, namely, $e^{\mathrm{w}}\left(\mathcal{H}_{\text {wav }}, n\right) / e^{\mathrm{r}}\left(\mathcal{H}_{\text {wav }}, n\right)$ is of the order $n^{1 / 2}[\log n]^{(s-1) / 2}$. 


\section{Discussion}

The parameter $\alpha$ in (25) describes how fast the Haar wavelet coefficients decay to zero as the resolution increases. This parameter also determines the convergence rate of scrambled net quadrature in the worst-case, random-case and average-case. Specifically, an integrand whose Haar wavelet coefficients decay rapidly will be integrated well by scrambled nets. Thus, in what follows let $\mathcal{H}_{\text {wav }, \alpha}=\mathcal{H}_{\text {wav }}$ to show this dependence explicitly.

The connection between smoothness of an integrand and the decay rate of its Haar wavelet coefficients has been explored in [HH99, HY00. Let $\mathcal{H}_{p}$ denote the Hilbert space of functions whose mixed partial derivatives of order up to $p$ in each coordinate are square integrable. It was shown in HH99, HY00 that $\mathcal{H}_{1}$ is a subspace of $\mathcal{H}_{\text {wav }, \alpha}$ for $\alpha<1$, and $\mathcal{H}_{2}$ is a subspace of $\mathcal{H}_{\text {wav }, \alpha}$ for $\alpha<3 / 2$. The spaces $\mathcal{H}_{p}$ for $p>2$ are only subspaces of $\mathcal{H}_{\text {wav }, \alpha}$ for $\alpha<3 / 2$, as can be seen by looking at the function $f(x)=x$. The problem of changing bases for Walsh series is discussed by [LP99].

In HH99, HY00] it was shown that the worst-case errors using scrambled nets for $\mathcal{H}_{1}$ and $\mathcal{H}_{2}$ are the same as for $\mathcal{H}_{\text {wav }, 1}$ and $\mathcal{H}_{\text {wav }, 3 / 2}$, respectively, even though the former spaces are a bit larger than the latter. The random-case errors using scrambled nets for $\mathcal{H}_{1}$ and $\mathcal{H}_{2}$ are $\mathrm{O}\left(n^{-1+\epsilon}\right)$ and $\mathrm{O}\left(n^{-3 / 2+\epsilon}\right)$, respectively, for any $\epsilon>0$. Whether $\epsilon=0$ is possible is an open question.

Although the base $b$ does not affect the convergence rate, it does affect the definition of the space $\mathcal{H}_{\text {wav, } \alpha}$. Consider the function $f(x)=1_{[0, a)}(x)$, where $a \in$ $[0,1)$. If $a$ has a terminating expansion in powers of $b^{-1}$, then $f(x) \in \mathcal{H}_{\mathrm{wav}, \alpha}$ for all $\alpha \geq 0$. However, if $a$ does not have a terminating expansion in powers of $b^{-1}$, then $f(x) \in \mathcal{H}_{\text {wav }, \alpha}$ only for $\alpha<1 / 2$.

Haar wavelets are related to Walsh series. The effectiveness of $(t, m, s)$-nets for integrating multivariate Walsh series has been studied by Larcher, Schmid, and their collaborators LT94, LS95a, LS95b, LLNS96, LSW96]. These investigations considered only deterministic rules in the worst-case setting, and not the randomcase or average-case. The spaces of integrands studied were Banach spaces defined in terms of the decay rates of the Walsh series coefficients. These spaces are related to, but different from, the Hilbert spaces of integrands considered here. For the situation considered by these authors, certain digital nets give optimal convergence rates in the worst-case setting, but arbitrary nets may be sub-optimal, a distinction not arising in our analysis.

Entacher [Ent97, Ent98] studied the integration of multivariate Haar wavelets by $(t, m, s)$-nets in the worst-case setting. The spaces of Haar series he considered are analogous to the Banach spaces of Walsh series studied by Larcher, Schmid and others. His upper bound on the worst-case convergence rate for nets is similar to Theorem 8 Entacher's lower bound applies only to nets, whereas our lower bound is for arbitrary quadrature rules. Moreover, Entacher did not study random-case or average-case errors.

\section{REFERENCES}

[Ent97] K. Entacher, Quasi-Monte Carlo methods for numerical integration of multivariate Haar series, BIT 37 (1997), 846-861. MR 98m:65011

[Ent98] K. Entacher, Quasi-Monte Carlo methods for numerical integration of multivariate Haar series II, BIT 38 (1998), 283-292. MR 99h:65010 
[Hei93] S. Heinrich, Random approximation in numerical analysis, Functional analysis. Proceedings of the Essen Conference, held in Essen, Germany, November 24 - 30, 1991. (K. D. Bierstedt, A. Pietsch, W. M. Ruess, and D. Vogt, eds.), Lecture Notes in Pure and Applied Mathematics, vol. 150, Marcel Dekker, New York, 1993, pp. 123-171. MR 94i: 65015

[HH99] F. J. Hickernell and H. S. Hong, The asymptotic efficiency of randomized nets for quadrature, Math. Comp. 68 (1999), 767-791. MR 99i:65021

[Hic96] F. J. Hickernell, The mean square discrepancy of randomized nets, ACM Trans. Model. Comput. Simul. 6 (1996), 274-296.

[HW01] F. J. Hickernell and H. Woźniakowski, The price of pessimism for multidimensional quadrature, J. Complexity 17 (2001), 625-659.

[HY00] F. J. Hickernell and R. X. Yue, The mean square discrepancy of scrambled $(t, s)-$ sequences, SIAM J. Numer. Anal. 38 (2000), 1089-1112. MR 2002c:65009]

[Lar98] G. Larcher, Digital point sets: Analysis and applications, Random and Quasi-Random Point Sets (P. Hellekalek and G. Larcher, eds.), Lecture Notes in Statistics, vol. 138, Springer-Verlag, New York, 1998, pp. 167-222. MR 99m:11085

[LLNS96] G. Larcher, A. Lauss, H. Niederreiter, and W. Ch. Schmid, Optimal polynomials for $(t, m, s)$-nets and numerical integration of Walsh series, SIAM J. Numer. Anal. 33 (1996), 2239-2253. MR 97m:65046

[Loh01] W.-L. Loh, On the asymptotic distribution of scrambled net quadrature, 2001, submitted for publication.

[LP99] G. Larcher and G. Pirsic, Base change problems for generalized Walsh series and multivariate numerical integration, Pac. J. Math. 189 (1999), 75-105. MR 2000f:42017

[LS95a] G. Larcher and W. Ch. Schmid, Multivariate Walsh-series, digital nets, and quasiMonte Carlo integration, In Niederreiter and Shiue [NS95], pp. 252-262. MR 97j:65002

[LS95b] G. Larcher and W. Ch. Schmid, On the numerical integration of high dimensional multivariate Walsh-series by quasi-Monte Carlo methods, Math. Comput. Simul. 38 (1995), 127-134. MR 96c:65009

[LSW96] G. Larcher, W. Ch. Schmid, and R. Wolf, Quasi-Monte Carlo methods for the numerical integration of multivariate Walsh series, Mathl. Comput. Modelling 23 (1996), 55-67. MR 97j:65044

[LT94] G. Larcher and C. Traunfellner, On the numerical integration of Walsh series by number-theoretic methods, Math. Comp. 63 (1994), 277-291. MR 94j:65030

[Nie92] H. Niederreiter, Random number generation and quasi-Monte Carlo methods, CBMSNSF Regional Conference Series in Applied Mathematics, SIAM, Philadelphia, 1992. MR 93h:65008

[Nov88] E. Novak, Deterministic and stochastic error bounds in numerical analysis, Lectures Notes in Math., no. 1349, Springer-Verlag, Berlin, 1988. MR 90a:65004

[NP01] H. Niederreiter and G. Pirsic, The microstructure of $(t, m, s)$-nets, J. Complexity 17 (2001), 683-696

[NS95] H. Niederreiter and P. J.-S. Shiue (eds.), Monte Carlo and quasi-Monte Carlo methods in scientific computing, Lecture Notes in Statistics, vol. 106, Springer-Verlag, New York, 1995. MR 97j:65002

[Owe95] A. B. Owen, Randomly permuted $(t, m, s)$-nets and $(t, s)$-sequences, In Niederreiter and Shiue NS95, pp. 299-317. MR 97k:65013

[Owe97a] A. B. Owen, Monte Carlo variance of scrambled net quadrature, SIAM J. Numer. Anal. 34 (1997), 1884-1910. MR 98h:65006

[Owe97b] A. B. Owen, Scrambled net variance for integrals of smooth functions, Ann. Stat. 25 (1997), 1541-1562. MR 98j:65004

[Owe98] A. B. Owen, Scrambling Sobol' and Niederreiter-Xing points, J. Complexity 14 (1998), 466-489. MR 2000c:65005

[Owe00] A. B. Owen, Monte Carlo, quasi-Monte Carlo, and randomized quasi-Monte Carlo, Monte Carlo and Quasi-Monte Carlo Methods 1998 (H. Niederreiter and J. Spanier, eds.), Springer-Verlag, Berlin, 2000, 86-97. MR 2002e:65013

[Rit00] K. Ritter, Average-case analysis of numerical problems, Lecture Notes in Mathematics, vol. 1733, Springer-Verlag, Berlin, 2000. MR 2001i:65001

[YH01] R. X. Yue and F. J. Hickernell, Integration and approximation based on scramble sampling in arbitrary dimensions, J. Complexity 17 (2001), 881-897. 
[YH02] R. X. Yue and F. J. Hickernell, The discrepancy and gain coefficients of scrambled digital nets, J. Complexity 18 (2002), 135-151.

[YM99] R. X. Yue and S. S. Mao, On the variance of quadrature over scrambled nets and sequences, Statist. Probab. Lett. 44 (1999), 267-280. MR 2000i:65008

[Yue99] R. X. Yue, Variance of quadrature over scrambled unions of nets, Statist. Sinica 9 (1999), 451-473. MR 2000h:65022

FB Informatik, Universität Kaiserslautern, PF 3049, D-67653 Kaiserslautern, GerMANY

E-mail address: heinrich@informatik.uni-kl.de

Department of Mathematics, Hong Kong Baptist University, Kowloon Tong, Hong KONG SAR, CHINA

E-mail address: fred@hkbu.edu.hk

College of Mathematical Science, Shanghai Normal University, 100 Guilin Road, Shanghai 200234, China

E-mail address: rxyue@online.sh.cn 